PoS $\quad \begin{aligned} & \text { PROCEEDINGS } \\ & \text { OF SCIENCE }\end{aligned}$

\title{
Can we improve on the Performance of Coded Mask Instruments?
}

\author{
Niels Lund* \\ National Space Center. Technical University of Denmark \\ E-mail: nl@space.dtu.dk
}

\begin{abstract}
All coded mask instruments flying today have analysis problems related to coding noise, due to incomplete sampling of the mask pattern or related to ghost images due to repeated copies of the coding pattern in the physical masks. Additionally, background photons arriving from the partially coded field of view is often the dominant source of image background. Methods to reduce or eliminate these problems are discussed.
\end{abstract}

7th INTEGRAL Workshop

September 8-11 2008

Copenhagen, Denmark

${ }^{*}$ Speaker. 


\section{Problems with current instrument designs}

All coded mask cameras flying today have analysis problems related to one or several of the following effects:

1. Coding noise,arising from an incomplete sampling of the coding pattern.

2. Ghost images due to the use of repeated copies of the coding pattern in the physical masks.

3. Background photons from outside the fully coded field of view.

Ideally we only want sky photons from within the fully coded field to arrive at the detector. But without collimation more than half of the photons may arrive from the partially coded field. Of course these are not only background photons - but sources in the partially coded field are more difficult to analyze because of the incomplete coding. A classical collimator in front of the detector can limit the background, but also interferes badly with the image coding.

The coding problem is the following: In order to realize the ideal constant value for the correlation between any two source shadowgrams it is mandatory that each shadowgram exactly covers the full mask pattern. If only some fraction of the mask pattern is illuminating the detector the resulting decoded image will exhibit systematic noise peaks which will adversely affect the limiting sensitivity.

In practice the full coverage of the mask has been achieved in many instrument designs by repeating the the basic pattern four times in the physical mask. (this is done for instance in the IBIS instrument on INTEGRAL [1] Fig. 1) The detector is sized to cover the full mask pattern only once. This configuration allows the detector to collect shadowgrams corresponding to complete mask patterns across a finite field of view. However the fact that the basic pattern is repeated in the physical mask implies that several strong 'ghost' sources appear in the reconstructed image for every real source observed, Fig. 2. Thus the image reconstruction is still far from ideal - and most of the photons anyway arrive from the partially coded sky regions.

Other designs, such as the Wide Field Camera flown on SAX [2], have avoided the ghost image problem by using only a single instance of the basic pattern in the mask and a detector of the same size Fig. 3 . But with this design it is only possible to sample the full pattern for an on-axis

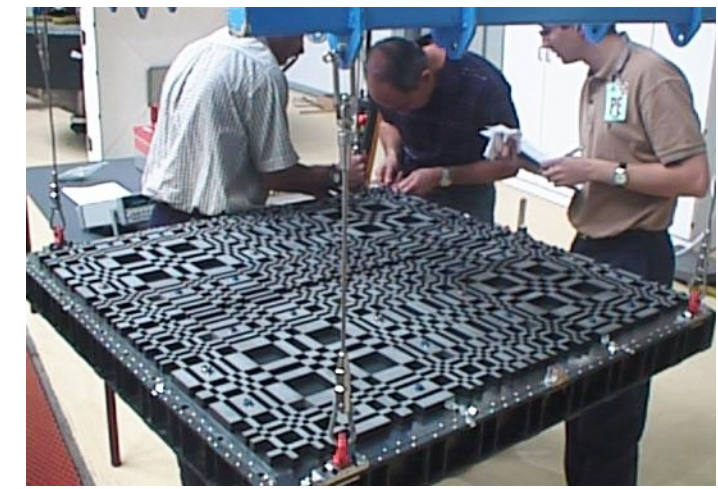

Figure 1: The IBIS mask containing four repeats of the basic pattern

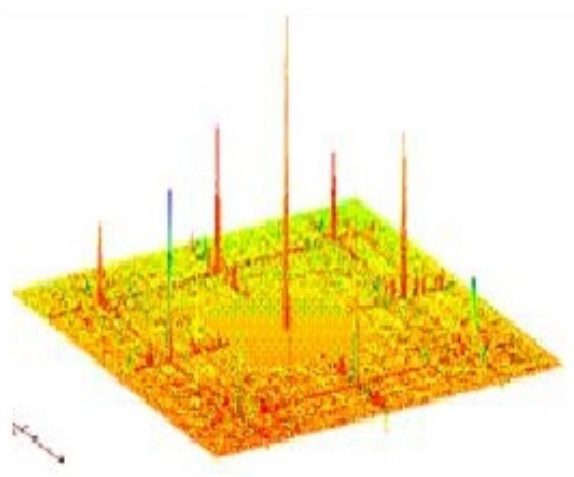

Figure 2: A raw IBIS image exhibiting ghost images of the central source 
source. Thus, apart from the singularity on-axis, the coding is incomplete for the full field of view. Serious vignetting of the image is inherent in this design. SPI [3] and JEM-X [4] on INTEGRAL are both limited by incomplete sampling everywhere in the image, and JEM-X also by collimator vignetting.

Despite such limitations these non ideal coded mask instruments have in fact yielded excellent results, but the aim of this work is to see if the above limitations are fundamental, or could maybe be alleviated by alternative designs.

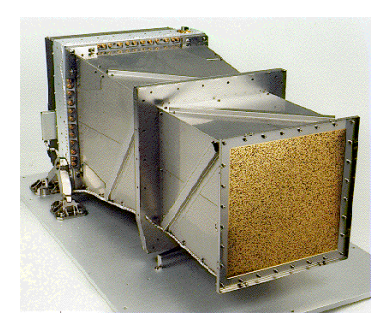

Figure 3: The SAX Wide Field Camera in which the mask and the detector were of the same size

\section{The new 'Quarterback' Configuration}

A system which assures perfect coding over a finite field of view without allowing ghost images to appear is illustrated in Fig. 4 and can be constructed with the following procedure:

1. Divide an ideal cyclic residue mask pattern (see section 3 ) into four quarters.

2. Through cyclic permutations of rows and columns make four new patterns each of which is centered on a different quarter of the original pattern.

3. Let 4 independent detectors, each of the size of one quarter pattern observe the sky through these four masks. The detector size is indicated by the red squares in the figures.

Then for every direction in the fully coded field the combined images from the four detectors will cover the original pattern exactly once, thus we have perfect coding! There will be no ghosts in the image because each detector is only seeing one code pattern.

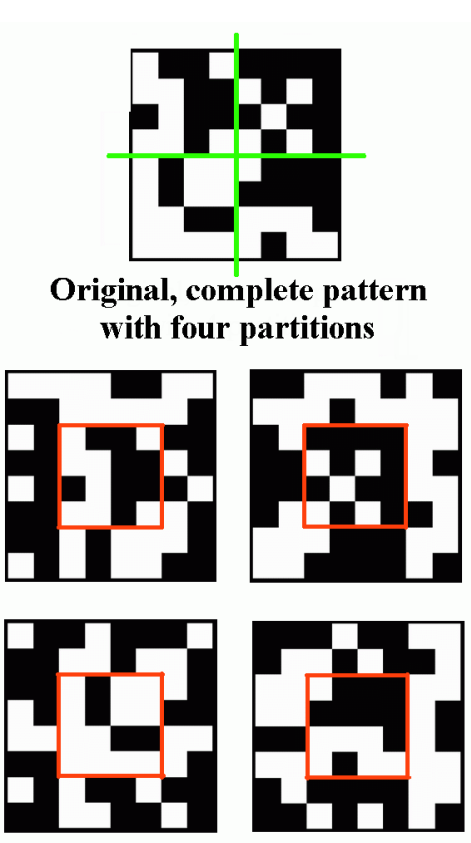

Four cyclically shifted patterns

Figure 4: 'Quarterback' Construction 


\section{Choice of code pattern}

\subsection{Code patterns}

Dicke, who was the inventor of the 'Scatter Hole Camera' [5], originally proposed a random arrangement of holes in the mask. But it was realized by Miyamoto [6] and by Fenimore and Cannon [7] that the mathematical methods developed for the study of cyclic difference sets (see Baumert (1971) [8]) could be used to make cameras with significantly better performance. With a random mask, the illumination of the detector will exhibit systematic variations according to the statistical fluctuations of the number of open cells in the mask for different illumination directions. This can be compensated for in the analysis without too much trouble. What is worse is that the correlation between the illumination patterns corresponding to different directions is also statistically fluctuating and this introduces noise in the image which depends sensitively on the relative brightness and locations in the image field of the sources to be imaged.

The 'uniformly redundant' patterns proposed by Miyamoto and by Fenimore and Cannon are 'ideal' in the sense that the number of transparent holes is constant for all directions, and the correlation between any two illumination patterns corresponding to two different directions is also perfectly constant. There is one important catch, unfortunately: the detector must sample the entire pattern once and only once! In practice this has been difficult to realize without repeating the same pattern four times in the mask - as it has been done for the IBIS instrument on INTEGRAL. Also, in practice, there are always some directions outside the central part of the field of view which do not project a full mask pattern on the detector, and these directions will be incompletely coded and will introduce systematic noise in the reconstructed images.

This work concerns the first of these two issues, the second one is harder to avoid.

\subsection{Size of code pattern}

One may have expected that the construction of the quarterback masks demanded that the length, $N_{\text {basic }}$, of the cyclic residue set could be factorized such that the set would exactly fit into a rectangle without surplus or missing elements. Fortunately this is not the case. The set must be chosen to exactly fit or have a few elements less than required. The excess elements, $N_{\text {excess }}$, in the mask rectangle should be filled with the first elements repeated, i.e. one should just keep counting modulo the set length. This will introduce noise of the order:

$$
\frac{\sqrt{N_{\text {excess }}}}{N_{\text {basic }}}
$$

which is insignificantly small once we are dealing with masks with many thousand elements and the number of excess elements are kept to a few tens.

\subsection{Mask transparency}

Another issue is the choice of mask transparency. Cyclic residue sets can be constructed with transparencies $1 / \mathrm{M}$, where $\mathrm{M}$ is an integer. Transparencies of $50 \%, 33 \%$ and $25 \%$ have been used in the past. It is a common misunderstanding that a transparency of $50 \%$ will achieve the best sensitivity. But this is only the case if the instrumental background is dominating over the diffuse sky background. Instruments for which the background is dominated by diffuse sky photons 
entering through the mask will achieve the highest sensitivity for weak sources with mask transparencies around 35\%, see f.i. Palmieri, (1974) [9], Gunson and Polychronopoulous, (1976) [10] and in't Zand et al., (1994) [11].

If the internal background of the detector is dominating then a higher transparency mask (up to $50 \%$ ) will be preferable. In photon limited applications such as for the study of short duration, intense bursts a higher mask transparency is also advantageous.

\section{Collimation}

In coded mask applications collimation may serve two purposes: it can reduce the coding noise arising from the partially coded field, and it will also improve the sensitivity within the fully coded field simply through the reduction of the diffuse flux from the surrounding sky regions. We shall discuss three type of collimation:

- detector collimation

- code mask collimation

- blinker collimation

\subsection{Detector collimation}

Classical collimators have always been detector collimators - it is the detector which must be protected against unwanted photons. The JEM-X detectors on INTEGRAL is one example. But in conjunction with a coded mask this type of collimation is quite unpleasant because it interferes with the coding process by blocking the signal from some of the open mask elements. This is the case if the pitch of the collimator is comparable to or larger than the mask cell dimensions as is the situation for JEM-X. In order to avoid an increase in the coding noise the pitch of the collimator must be smaller than the cell dimension. This will normally be a very fine collimator which will be a challenge to construct, and which may not be effective for energies much above 50 $\mathrm{keV}$. Additionally, such a fine collimator will not be mechanically strong, and it cannot serve the purpose of a support for the window of a gas detector. But for solid state detectors designed for low energies it may work.

\subsection{Code mask collimation}

Alternatively one can use the pattern of the coded mask itself as the basis for the collimation. This may be done in two ways: by the use of a thick mask or by the use of masks with several layers

In a thick mask the thickness, $\mathrm{T}$, of the obscured mask elements is comparable to the dimensions of the mask cells, $\mathrm{C}$. Examples of such masks are the IBIS (T/C $=16 / 11.2=1.5)$ and SPI $(\mathrm{T} / \mathrm{C}=30 / 60=0.5$ ) masks on INTEGRAL. For comparison for JEM-X we have $\mathrm{T} / \mathrm{C}=0.5 / 3.3=0.15$. With a thick mask there is a collimation effect build into the mask itself. However the collimation is obviously uneven, because isolated open cells will be more strongly collimated than clusters of adjacent open cells. The uneven collimation contribute to the noise in the images. 
The use of multi layer masks gives the extra freedom of using different hole sizes (but of course the same pattern!) in the different mask layers. This allows to design mask systems where there is almost no interference between the coding and the collimation - unfortunately only a very limited collimation effect (a 20\% reduction in the diffuse flux) can be achieved in this way, so it is not a really interesting option. Thus there seem to be no way one can avoid to introduce coding noise also when using mask collimation.

\subsection{Blinker collimation}

The last collimation type to be discussed is the most primitive one, just to equip the detectormask assembly with blinkers - a long tube shielding off the unwanted sky regions. The blinker technique is effective, but rather impractical due to the required size of the shielding tube. But given that with the Quarterback detector-mask configuration it now appears possible to achieve sky images with minimal coding noise it may be worthwhile to take a fresh look at ways to deploy blinkers around coded mask systems after launch.

\section{An important observation}

On the last day of the workshop Dr. C. Wunderer pointed out to me that the quarterback construction carries with it an unpleasant limitation: Either the field-of-view of the quarterback instrument is only one quarter of the field of view a corresponding 'IBIS'-construction (a full detector observing through a mask with four repeated patterns), or the angular resolution of the quarterback construction is only half of that of the IBIS-type instrument.

Both of these limitations are very serious and probably makes the application of the simple quarterback construction as described above unattractive for practical instruments.

\section{Conclusions}

A novel mask and detector configuration, 'Quarterback', for coded mask instruments have been presented. This configuration will eliminate two of the three systematic noise sources which have been present in past instruments, of this type, namely coding noise and ghost images (within the fully coded field).

However the objections raised by Dr. Wunderer (see section 5) brings this study back to its starting point, which was a search for mathematical sets which could provide noise free coding even with incomplete sampling. The quarterback construction might turn out to be useful if such mathematical sets can be found, because the quarterback construction guarantees that a constant fraction of the mask elements enters into the imaging process.

Different collimation techniques have been considered to see if it is possible to reduce the noise signals coming from the partially coded field. No perfect solution has been found, but it is worth stressing that the use of the quarterback configuration together with an IBIS type 'thick mask' should result in an instrument with a response function identical to that of IBIS, but free of image ghosts 


\section{Acknowledgments}

The author is indebted to Cornelia Wunderer, Space Sciences Laboratory, UC Berkeley; Jørn Justesen, DTU-Fotonics, Daniel Gordon, IDA, La Jolla; Søren Brandt and Nicolai Brejnholt, DTUSpace for useful discussions and assistance. This work was initiated during a stay at the Sabanci University, Turkey supported by the ASTRONS project under the European Commission, Marie Curie Program.

\section{References}

[1] Ubertini, P., Lebrun, F., Di Cocco, G., Bazzano, A., Bird, A. J., Broenstad, K., Goldwurm, A., La Rosa, G., Labanti, C., Laurent, P., Mirabel, I. F., Quadrini, E. M., Ramsey, B., Reglero, V., Sabau, L., Sacco, B., Staubert, R., Vigroux, L., Weisskopf, M. C., and Zdziarski, A. A., "IBIS: The Imager on-board INTEGRAL," A\&A 411, L131-L139 (Nov. 2003).

[2] Boella, G., Butler, R. C., Perola, G. C., Piro, L., Scarsi, L., and Bleeker, J. A. M., "BeppoSAX, the wide band mission for X-ray astronomy," AAPS 122, 299-307 (Apr. 1997).

[3] Vedrenne, G., Roques, J.-P., Schönfelder, V., Mandrou, P., Lichti, G. G., von Kienlin, A., Cordier, B., Schanne, S., Knödlseder, J., Skinner, G., Jean, P., Sanchez, F., Caraveo, P., Teegarden, B., von Ballmoos, P., Bouchet, L., Paul, P., Matteson, J., Boggs, S., Wunderer, C., Leleux, P., Weidenspointner, G., Durouchoux, P., Diehl, R., Strong, A., Cassé, M., Clair, M. A., and André, Y., “SPI: The spectrometer aboard INTEGRAL," A\&A 411, L63-L70 (Nov. 2003).

[4] Lund, N., Budtz-Jørgensen, C., Westergaard, N. J., Brandt, S., Rasmussen, I. L., Hornstrup, A., Oxborrow, C. A., Chenevez, J., Jensen, P. A., Laursen, S., Andersen, K. H., Mogensen, P. B., Rasmussen, I., Omø, K., Pedersen, S. M., Polny, J., Andersson, H., Andersson, T., Kämäräinen, V., Vilhu, O., Huovelin, J., Maisala, S., Morawski, M., Juchnikowski, G., Costa, E., Feroci, M., Rubini, A., Rapisarda, M., Morelli, E., Carassiti, V., Frontera, F., Pelliciari, C., Loffredo, G., Martínez Núñez, S., Reglero, V., Velasco, T., Larsson, S., Svensson, R., Zdziarski, A. A., Castro-Tirado, A., Attina, P., Goria, M., Giulianelli, G., Cordero, F., Rezazad, M., Schmidt, M., Carli, R., Gomez, C., Jensen, P. L., Sarri, G., Tiemon, A., Orr, A., Much, R., Kretschmar, P., and Schnopper, H. W., "JEM-X: The X-ray monitor aboard INTEGRAL," A\&A 411, L231-L238 (Nov. 2003).

[5] Dicke, R. H., "Scatter hole cameras for X-rays and gamma rays," ApJL 153, L101-L106 (1968).

[6] Miyamoto, S., “Hadamard transform x-ray telescope,” S.Sci.Instr. 3, 473-481 (1977).

[7] Fenimore, E. E. and Cannon, T. M. Appl.Opt. 17, 337 (1978).

[8] Baumert, L. D., [Lecture Notes in Mathematics No 1822: Cyclic Difference Sets], Springer, Berlin (1971).

[9] Palmieri, T. M., “An x-ray telescope sensitive at high energy," AphSpSci 20, 431-445 (1974).

[10] Gunson, J. and Polychronopoulous, B., "Optimum design of a coded mask x-ray telescope for rocket applications," MNRAS 177, 485 (1976).

[11] in't Zand, J. J. M., Heise, J., and Jager, R., "The optimum open fraction of coded apertures ...," A\&A 288, 665-674 (1994). 